\title{
Test and Analysis on the Interference to the CBTC Systems by Wi- Fi Signals
}

\author{
Li Ming ${ }^{1}$, Wang Hua-shen ${ }^{1}$, Zhao Hong-li ${ }^{2}$ and $\mathrm{Zhu} \mathrm{Li}^{2}$ \\ ${ }^{1}$ National Research Centre of Rail Transit Safety Assessment, Beijing 100044, \\ China \\ ${ }^{2}$ State Key Laboratory of Rail Traffic Control and Safety, Beijing Jiaotong \\ University, Beijing 100044, China \\ 13111046@bjtu.edu.cn
}

\begin{abstract}
Communication-Based Train Control (CBTC) system is an automated train control system using bidirectional train-ground communications to ensure the safe operation of rail vehicles. The interference from Wi-Fi signals to the CBTC signals has significant impacts on the train control performance. Most of previous works use data from simulation platform. This paper proposes a method to test the Wi-Fi interference to the Communication Based Train Control(CBTC) systems. The test is made mainly based on real subway, and supplemented by laboratory simulation platform. The collected data is to be compared and analyzed. The test result shows that the signal transmission mode of waveguide is the most reliable, followed by the leaky cable. The transmission mode of free wave has the worst anti-interference capability to Wi-Fi signals. This is because that both the waveguide and leaky cable transmission have a better encapsulation compared with the free space transmission. Also applying for specific bands can effectively reduce the interference transferred from Wi-Fi signals to the CBTC.
\end{abstract}

Keywords: Wi-Fi; CBTC; free wave; leaky cable; waveguide

\section{Introduction}

The CBTC (Communication Based Train Control) [1] signal interrupts caused by interference influence the security and effectiveness of the CBTC systems, which may seriously reduce the operational efficiency of subway. Also, if the time of signal interrupts is long enough, the emergency brake is needed which can not only waste energy but also pose a potential threat to the safety of passengers leading to passengers' tumbling or even getting hurt. Thus it is important to solve the problem of interference exists in Shenzhen subway signal system.

Since July 2012, the train in the Shekou line and midline of Shenzhen subway was caused to several emergency stops due to interference to signaling system [2,3]. The frequent occurrences have caused a great deal of security risks which sparked common concerns.

According to the fault location test made by Shenzhen subway and local radio administration, the command signal on subway is abnormal when the portable $\mathrm{Wi}-\mathrm{Fi}$ routers carried by passengers are working while get right when the routers are closed. Therefore, the conclusion given by the administration is that the wireless devices interfere the communication signals transmitted from subway to Zone Controller (ZC) [4], resulting in mistaken signals and emergency stops. But Shenzhen subway and local radio administration only show us the cause of the CBTC signal interruption and they have not analysed in depth.

At present, the wireless communication from subway to ZC in CBTC systems generally use $2.4 \mathrm{GHz}$ frequency band, which is as same as common WLAN devices. 
Once passengers open their Wi-Fi routers, the subway communication signals will be interfered. According to [5, 6], the subway operator can not ban Wi-Fi routers, so we can focus on the anti-interference performances of CBTC signals to Wi-Fi signals.

Although some works have been done to address the interference problem in the paper [7], it is not enough. CBTC signals generally can be transmitted in three ways: free wave, leaky cable and waveguide $[8,9]$. The testing method for the roof antenna is not intact, and there is not a testing method for the waveguide.

In this paper, we add another scenario in the testing method for the roof antenna and propose a testing method for the waveguide. Also the testing data are collected and analysed. Based on the testing results, the anti-interference performances of CBTC signals transmitted in three different ways are contrasted which can be strong support for solving the problem of the anti-interference performances of CBTC signals to Wi-Fi signals.

\section{The Impact of Signal Breaking Off to CBTC Systems}

With the rapid development of wireless communication technology, people began to pay close attention to safety of CBTC systems. The outstanding advantage of CBTC systems is the bidirectional communication between train and $\mathrm{ZC}$ equipments by means of wireless communication medium, replacing the train control based on track circuit [8]. CBTC systems have the capacity of transmitting vast information in a short time and make moving automatic block easy to achieve [10]. CBTC signals generally can be transmitted in three ways: free wave, leaky cable and waveguide [8, 9].

Bidirectional communication is the key technique in CBTC systems. Once the CBTC signals are interrupted, it will lead to serious risks [11]. Signal interrupts can be devided into two kinds: communication delay and data packet loss, and both of them may influence the security and efficiency of CBTC systems [12]. Security refers to the capability for controlled system of avoiding potentially dangerous or unstable state. Efficiency means that in every communication period, the train and $\mathrm{ZC}$ equipments send the corresponding information to each other as soon as the received information is processed.

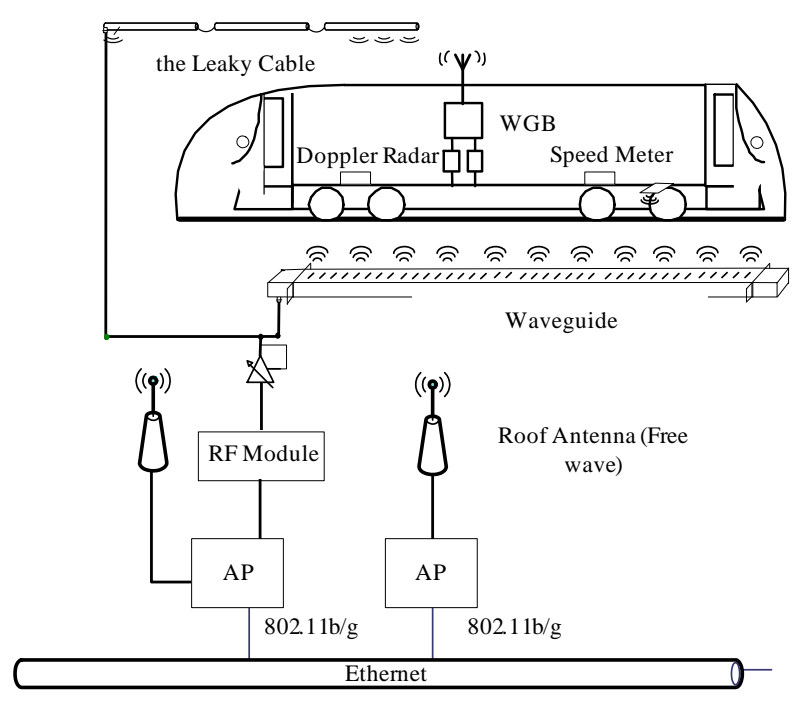

Figure 1. CBTC Signaling Schematic Plot

Figure 1 is CBTC signaling schematic plot. It shows us that three different ways for CBTC signals transmitting: free wave, leaky cable and waveguide. Free wave, 
leaky cable and waveguide should be linked to AP. CBTC signals transmitted in all the three ways should go to the Ethernet though the AP first and then to the $\mathrm{ZC}$.

\section{The Proposed Testing Methods}

Aiming at the situation that trains in Shenzhen Subway have been caused to emergency stops for the Wi-Fi signals' interference and considering the severe influence of signal breaking off to the train and passengers, we propose methods to test the anti-interference performances of CBTC signals to the Wi-Fi signals.

Definition 1. When the CBTC system transmitted in the frequency of $2412 \mathrm{MHz}$, we name it transmitted in the first channel. When $2417 \mathrm{MHz}$, the second channel ;When $2422 \mathrm{MHz}$, the third channel ;When $2427 \mathrm{MHz}$, the fourth channel ;When $2432 \mathrm{MHz}$, the fifth channel ;When $2437 \mathrm{MHz}$, the sixth channel ;When $2442 \mathrm{MHz}$, the seventh channel ;When $2447 \mathrm{MHz}$, the eighth channel ; When $2452 \mathrm{MHz}$, the ninth channel ;When $2457 \mathrm{MHz}$, the tenth channel ; When $2462 \mathrm{MHz}$, the eleventh channel .

Definition 2. When signals transmitted in the sixth channel the fifth and seventh channel are all it's first adjacent channel ,the fourth and eighth channel are all it's second adjacent channel and by this analogy.

The testing methods consist of three parts related to the signal transmission modes. The first part is to test the interference when CBTC signal is transmitted by free wave, then in leaky cable and finally in waveguide. At last the anti-interference performances of the three transmission modes will be contrasted.

The detailed testing steps are listed as below. Connect the CBTC system strictly and make sure it can work normally. Close other surrounding electronic equipments to lessen testing errors caused by the uncorrelated devices. Set the transmitting channel as the sixth channel, which equals to the transmitting frequency is $2437 \mathrm{MHz}$. Set the modulation mode as OFDM/DSSS and duty cycle as $100 \%$. Choose a suitable location and connect the coupler, the CBTC AP, roof antenna, leaky cable and waveguide. Test under the condition that there can be Wi-Fi signals interference to CBTC signals in the same channel or adjacent channel then record the frequency and transmitting power of Wi-Fi signals when CBTC signals breaking off.

\subsection{Testing Method for the AP Antenna(Free wave)}

\subsubsection{Testing the Interference to Roof AP Antenna}

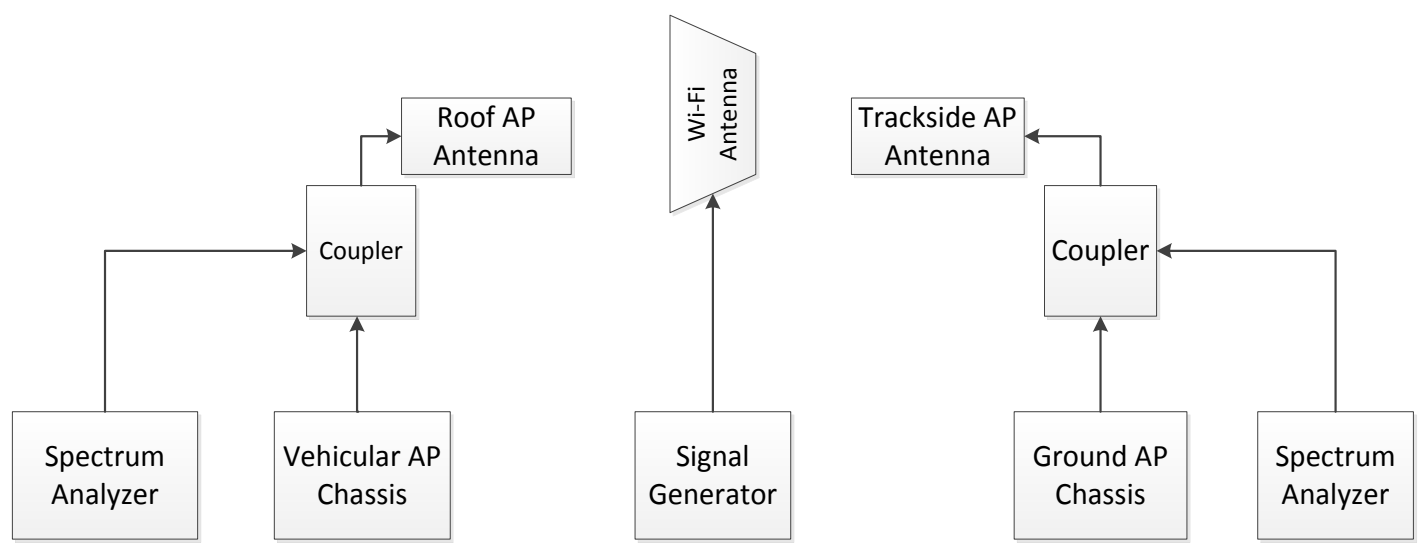

Figure 2. Schematic Interference Experiments On-board AP

As shown in Figure 2, connect couplers and spectrum analyzer. Make sure the CBTC system work properly, and set up the sixth channel as a communication 
channel for CBTC. Connect the spectrum analyzer with the roof AP antenna through the coupler, measure the effective command signal intensity and make sure it should be at least higher than the receiver sensitivity by $10 \mathrm{~dB}$. Under the circumstances, command signal can be transmitted normally.

When CBTC downlink signal is transmitted from trackside AP antenna to the roof AP antenna, equip Wi-Fi antenna directly facing to roof AP antenna on the same horizontal line. Keep the Wi-Fi antenna and roof AP antenna $10 \mathrm{~cm}$ apart and transmit signals in the opposite direction to insure the best interference effect [13]. Connect the Spectrum Analyzer with the interference source. Increase the transmitting power of interference signal source gradually until the CBTC system signal breaking off and record the interference signal intensity at that exact moment.

Adjust transmitting frequency of the interference signal to $2412 \mathrm{MHz}, 2417 \mathrm{MHz}$, $2422 \mathrm{MHz}, 2427 \mathrm{MHz}, 2432 \mathrm{MHz}, 2437 \mathrm{MHz}, 2442 \mathrm{MHz}, 2447 \mathrm{MHz}, 2452 \mathrm{MHz}$, $2457 \mathrm{MHz}$ and $2462 \mathrm{MHz}$. Repeat the same steps as mentioned and measure the critical interference signal power that interrupts the CBTC system respectively.

\subsubsection{Testing the Interference to Trackside AP Antenna}
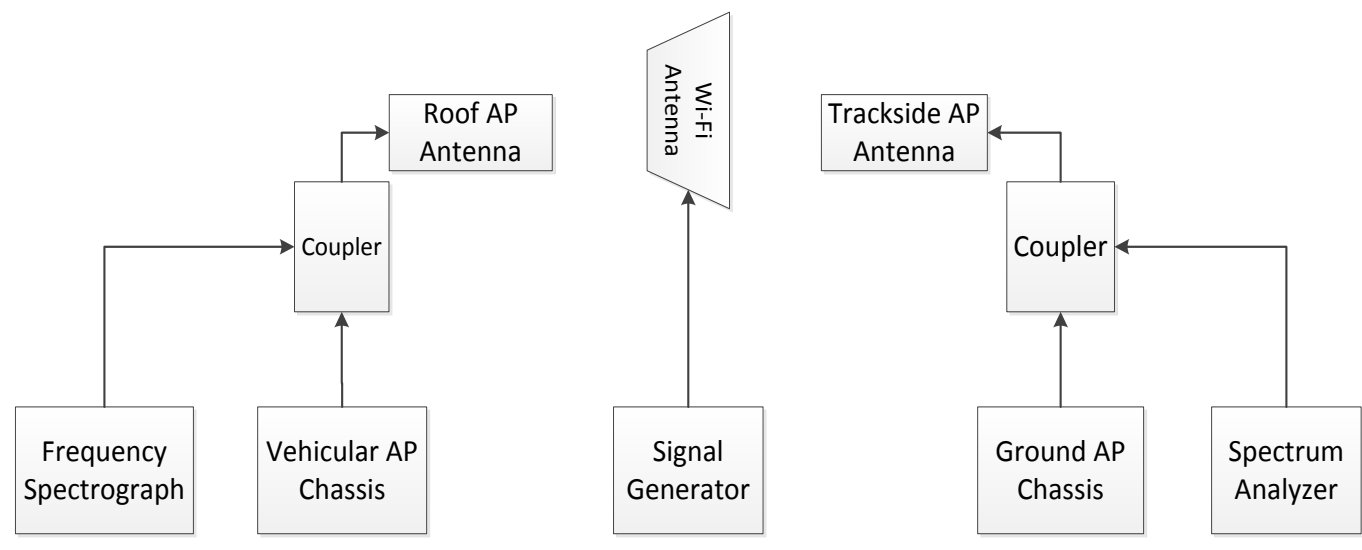

Figure 3. Testing Method for the Trackside Antenna (Free wave)

Connect all the components correctly shown in figure 3 and set CBTC transmitting channel as the sixth channel. Make sure the command signal intensity is higher than receiving sensitivity by $10 \mathrm{~dB}$.

When CBTC uplink signal is transmitted from roof AP antenna to the trackside AP antenna, equip Wi-Fi antenna directly facing to trackside AP antenna. Keep the WiFi antenna and the trackside AP antenna $10 \mathrm{~cm}$ apart and transmit signals in the opposite direction to insure the best interference effect [13]. Connect the Spectrum Analyzer with the interference source. Increase the transmitting power of interference signal source gradually until the CBTC system signal interrupted and record the interference signal strength at that exact moment.

Adjust transmitting frequency of the interference signal to $2412 \mathrm{MHz}, 2417 \mathrm{MHz}$, $2422 \mathrm{MHz}, 2427 \mathrm{MHz}, 2432 \mathrm{MHz}, 2437 \mathrm{MHz}, 2442 \mathrm{MHz}, 2447 \mathrm{MHz}, 2452 \mathrm{MHz}$, $2457 \mathrm{MHz}$ and $2462 \mathrm{MHz}$. Repeat the same steps as mentioned and measure the critical interference signal power that interrupts the CBTC system respectively. 


\subsection{Testing Method for the Leaky Cable}

Leaky Cable

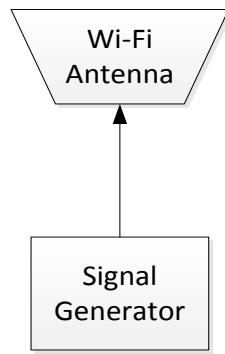

Figure 4. Testing Method for the Leaky Cable

Figure 4 shows the top view of the testing method for the leaky cable. The Wi-Fi antenna is placed at 2 meters below the leaky cable. In consideration of the actual situation, the wireless devices carried by passengers will not be less than 2 meters away from the leaky cable. The chosen distance may insure the best interference effect from Wi-Fi signals to the CBTC system [13].

Set up the sixth channel as a communication channel for CBTC and connect all the components correctly shown in figure 4. Make sure the command signal intensity is higher than receiving sensitivity by $10 \mathrm{~dB}$. Connect the Spectrum Analyzer with the interference source. Increase the transmitting power of interference signal source gradually until the CBTC system signal interrupted and record the interference signal strength at that exact moment.

Adjust transmitting frequency of the interference signal to $2412 \mathrm{MHz}, 2417 \mathrm{MHz}$, $2422 \mathrm{MHz}, 2427 \mathrm{MHz}, 2432 \mathrm{MHz}, 2437 \mathrm{MHz}, 2442 \mathrm{MHz}, 2447 \mathrm{MHz}, 2452 \mathrm{MHz}$, $2457 \mathrm{MHz}$ and $2462 \mathrm{MHz}$. Repeat the same steps as mentioned and measure the critical interference signal power that interrupts the CBTC system respectively.

\subsection{Testing Method for the Waveguide}
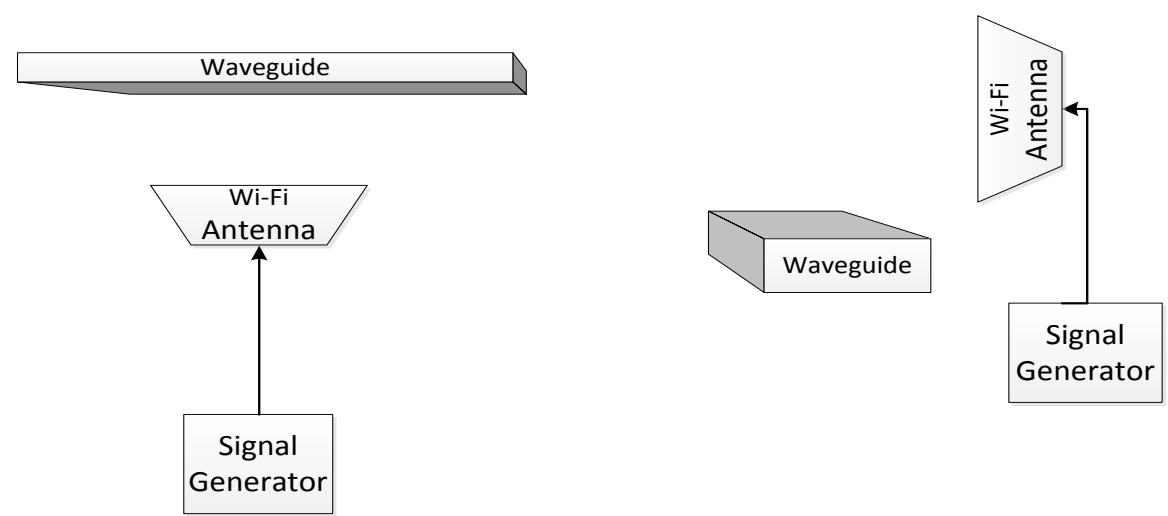

Figure 5. Testing Method for the Waveguide

Figure 5 shows the top view of the testing method for waveguide on the left and side view on the right. Place Wi-Fi antenna $45^{\circ}$ diagonally above the waveguide and make them $1 \mathrm{~m}$ apart in the horizontal direction. The chosen angle and distance are beneficial to imitate the minimum distance in reality between the wireless devices on 
train and the waveguide beside the track, which will insure the best interference effect [13].

Set CBTC transmitting channel as the sixth channel. Connect the Spectrum Analyzer with waveguide by a coupler and watch the real-time command signal to make sure the command signal intensity is higher than receiving sensitivity by $10 \mathrm{~dB}$. Increase the transmitting power of the interference signal source gradually until the CBTC system signal breaking off. Connect the Spectrum Analyzer with the interference source and record the interference signal strength at that exact moment [13].

Adjust transmitting frequency of the interference signal to $2412 \mathrm{MHz}, 2417 \mathrm{MHz}$, $2422 \mathrm{MHz}, 2427 \mathrm{MHz}, 2432 \mathrm{MHz}, 2437 \mathrm{MHz}, 2442 \mathrm{MHz}, 2447 \mathrm{MHz}, 2452 \mathrm{MHz}$, $2457 \mathrm{MHz}$ and $2462 \mathrm{MHz}$. Repeat the same steps as mentioned and measure the critical interference signal power that interrupts the CBTC system respectively.

\section{Testing Results and Analysis}

\subsection{Testing Results}

The CBTC system signals should be transmitted through the sixth channel, the signal transmitting power ranges from $10 \mathrm{~mW}$ to $100 \mathrm{~mW}$.

According to the national regulations, the signal transmitting power cannot exceed $100 \mathrm{~mW}$ in $2.4 \mathrm{GHz}$ frequency band, which means we can take no account of the interference above the power of $100 \mathrm{~mW}$. The conversion formula about $\mathrm{mW}$ and $\mathrm{dBm}$ is shown as equation (1):

$$
P^{\prime}=10 \lg P
$$

Where, $P$ is in the unit of $\mathrm{mW}$ and $P^{\prime}$ is in the unit of $\mathrm{dBm}$.

$$
P^{\prime}=10 \lg 100=20 \mathrm{~dB} \mathrm{~m}
$$

Based on equation (2), $20 \mathrm{dBm}$ is the max valid signal transmitting power in the unit of $\mathrm{dBm}$. Therefore, interference signals exceed $20 \mathrm{dBm}$ are not in the scope of discussion.

Table 1. Power of Interference Signals that Interrupt CBTC Signals by Free Wave

\begin{tabular}{|c|c|}
\hline Frequency (MHz) & Power $(\mathrm{dBm})$ \\
\hline 2412 & $>20$ \\
2417 & $>20$ \\
2422 & 8 \\
2427 & 6 \\
2432 & 4 \\
2437 & 5 \\
2442 & 4 \\
2447 & 6 \\
2452 & 8 \\
2457 & $>20$ \\
2462 & $>20$ \\
\hline
\end{tabular}

Table 1 shows the critical power of interference signals that interrupt CBTC signals transmitted by free wave. The first list is the frequency of the interference signal and the second list shows that crank up the power of interference signals until 
the CBTC system signals breaking off, it's the minimum power of interference signals leading to the CBTC system breaking off.

When interference signal is transmitted towards the roof AP antenna, it will reduce the SNR of downlink signals and result in signal interruptions. While interference signal is transmitted towards the trackside AP antenna, it will reduce the SNR of uplink signals and result in signal interruptions. The two interferences have the same principles, and the mass testing data indicate that the critical power of the interference signals in the same frequency are identical when signals to roof or trackside AP are interrupted.

Table 2. Power of Interference Signals that Interrupt CBTC Signals in the Leaky Cable

\begin{tabular}{|c|c|}
\hline Frequency (MHz) & Power $(\mathrm{dBm})$ \\
\hline 2412 & $>20$ \\
2417 & $>20$ \\
2422 & 11 \\
2427 & 7 \\
2432 & 5 \\
2437 & 6 \\
2442 & 5 \\
2447 & 7 \\
2452 & 11 \\
2457 & $>20$ \\
2462 & $>20$ \\
\hline
\end{tabular}

Table 2 shows the critical power of interference signals that interrupt CBTC signals transmitted in the leaky cable. The first list is the frequency of the interference signal and the second list shows that crank up the power of interference signals until the CBTC system signals breaking off, it's the minimum power of interference signals leading to the CBTC system breaking off. When the CBTC signal is transmitted in the leaky cable, the interference signal mainly has an impact on the process from the leaky cable openings to the train receiving end, which will reduce SNR of both the uplink and downlink signals and result in signal interruptions.

Table 3. Power of Interference Signals that Interrupt CBTC Signals in the Waveguide

\begin{tabular}{|c|c|}
\hline Frequency (MHz) & Power (dBm) \\
\hline 2412 & $>20$ \\
2417 & $>20$ \\
2422 & 12 \\
2427 & 8 \\
2432 & 5 \\
2437 & 6.5 \\
2442 & 5 \\
2447 & 8 \\
2452 & 12 \\
2457 & $>20$ \\
2462 & $>20$ \\
\hline
\end{tabular}


Table 3 shows the critical power of interference signals that interrupt CBTC signals transmitted in the waveguide. The first list is the frequency of the interference signal and the second list shows that crank up the power of interference signals until the CBTC system signals breaking off, it's the minimum power of interference signals leading to the CBTC system breaking off. When the CBTC signal is transmitted in the waveguide, the interference signal mainly influence the process from the waveguide openings to the train receiving end, which will reduce SNR of both the uplink and downlink signals and result in signal interruptions.

\subsection{Analyze: Anti-interference Performances Contrast}

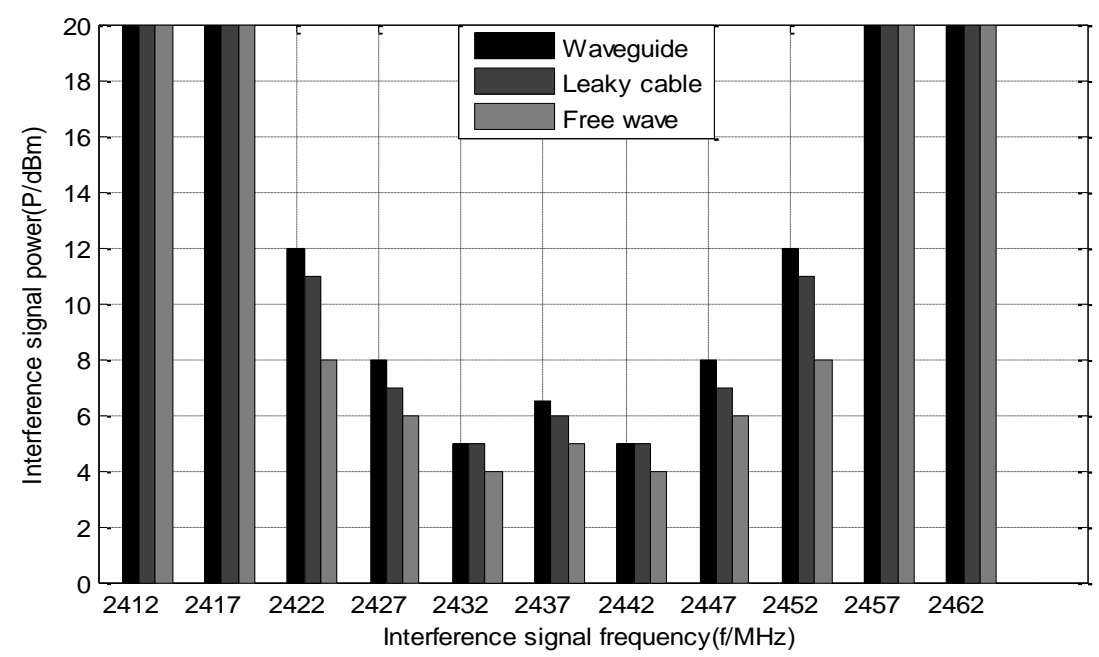

Figure 6. Anti-interference Performances Contrast of the Three Transmission Modes

Figure 6 shows us that the contrast of interference effect on CBTC signals transmitted in the three transmission modes. Horizontal coordinate represents the frequency of the interference signal and vertical coordinate means the critical power of the interference signal when CBTC signal is interrupted.

From the bar chart above we can see when the interference signal is transmitted in the third ,fourth ,sixth ,eighth and ninth channel ,it is clear that the minimum power of the interference signal interrupting the CBTC signals is the highest by waveguide and then by leaky cable ,it's the lowest by free wave. When it's transmitted in the fifth and seventh channel ,the minimum power of the interference signal interrupting the CBTC signals can be $5 \mathrm{dBm}$ for both waveguide and leaky cable, it is $4 \mathrm{dBm}$ for free wave .For these two channel ,the anti-interference capability of the CBTC signals to the Wi-Fi signals can be the same for the transmitting mode of waveguide and leaky cable, and both are better than that by free wave .

On the whole the interference signal power interrupting CBTC signals transmitted in waveguide is the highest, it ranges the second when transmitted by leaky cable and it is the lowest when transmitted by free wave.

The signal loss by the free wave is serious on account that it is transmitted to all directions, while the leaky cable and waveguide have the advantage of better encapsulation which is helpful for weakening the signal loss to a great extent .At the same time the ability of anti-interference can be stronger when CBTC system signal transmitted in closed-in spaces as thorough the leaky cable and waveguide.

It indicates that the signal transmission mode of waveguide is the most reliable, followed by the leaky cable. The transmission mode of free wave has the worst anti- 
interference capability to Wi-Fi signals. In other words, the anti-interference performance of CBTC signals transmitted in the leaky cable is better than free wave, while worse than waveguide.

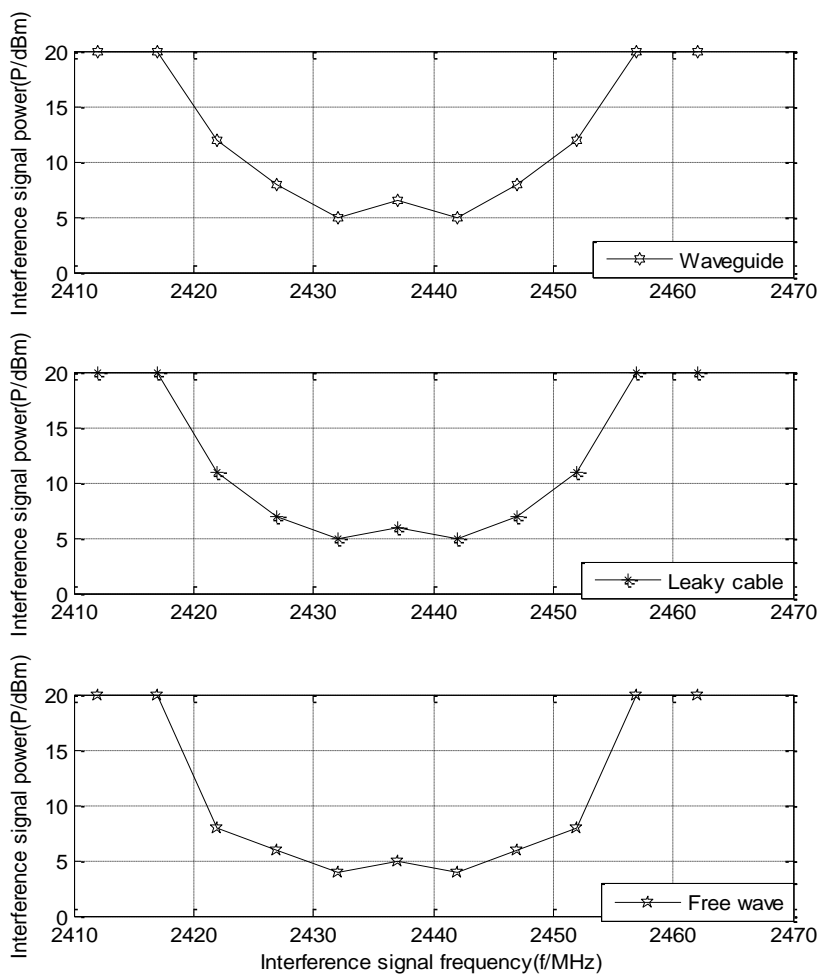

\section{Figure 7. Interference Effect Trend on CBTC Signals Transmitted in the Three Transmission Modes}

Figure 7 shows the interference effect trend on CBTC signals transmitted in the three transmission modes. Horizontal coordinate represents the frequency of the interference signal and vertical coordinate means the critical power of the interference signal when CBTC signal is interrupted.

When the CBTC system signals are transmitted in the sixth channel by waveguide and the interference signal is transmitted in the third channel ,the minimum power of the interference signal interrupting the CBTC signals can be $12 \mathrm{dBm}$ which is the same power as in the ninth channel. When it is in the fourth and eighth channel, the power can be the same $8 \mathrm{dBm}$; When it is in the fifth and seventh channel, the power can be the same $5 \mathrm{dBm}$. For leaky cable, when it is in the third and ninth channel, the power can be the same 11 $\mathrm{dBm}$; when it is in the fourth and eighth channel ,the power can be the same $7 \mathrm{dBm}$; when it is in the fifth and seventh channel ,the power can be the same $5 \mathrm{dBm}$.For free wave, when it is in the third and ninth channel, the power can be the same $8 \mathrm{dBm}$; when it is in the fourth and eighth channel ,the power can be the same $6 \mathrm{dBm}$; when it is in the fifth and seventh channel, the power can be the same $4 \mathrm{dBm}$.

It reveals that for a certain transmission mode, as long as the absolute values of frequency difference between interference and CBTC signals are equal, there will exists almost no difference in the interference effect.

From Figure 7 for all the three mode of transmission, under the condition that the CBTC system signals are transmitted in the sixth channel and the interference signal is transmitted in the first, second ,tenth and eleventh channel, when the power of the interference signal 
increases to $20 \mathrm{dBm}$, the CBTC signals can be transmitting normally .So interference signals in the fourth adjacent channel or above almost have no influence on the CBTC systems.

It is clear in Figure 7 that for waveguide when the interference signal is transmitted in the fifth and seventh channel, the minimum power of the interference signal interrupting the CBTC signals can be $5 \mathrm{dBm}$, but when it is in the six channel, the power can be 6.5 $\mathrm{dBm}$. For leaky cable when the interference signal is transmitted in the fifth and seventh channel, the minimum power of the interference signal interrupting the CBTC signals can be $5 \mathrm{dBm}$, but when it is in the six channel, the power can be $6 \mathrm{dBm}$. For free wave when the interference signal is transmitted in the fifth and seventh channel, the minimum power of the interference signal interrupting the CBTC signals can be $4 \mathrm{dBm}$, but when it is in the six channel ,the power can be $5 \mathrm{dBm}$. So interference signals in the first adjacent channel have the most serious influence, even than the co-channel signals.

From the analysis above, although the performance for CBTC signals transmitted by waveguide is better, there can still be signal interruptions when CBTC signals are influenced by the interference signal .While interference signals in the fourth adjacent channel or above almost have no influence on the CBTC systems. Thus improving antiinterference capacity cannot solve the problem that the interference from Wi-Fi signals to CBTC systems essentially, applying for specific frequency bands is much more efficient.

\section{Conclusion and Future Work}

This paper proposes a method to test the Wi-Fi interference to the CBTC signals transmitted by free wave, leaky cable and waveguide. Then the anti-interference performances of the three transmission modes are compared and analyzed which can be a good reference to the railway administration. In the future studies, we will focus on more about why and how there can be different performance of anti-interference for different transmission mode.

\section{References}

[1] R. D. Pascoe and T. N. Eichorn, "What is communication-based train control?", Vehicular Technology Magazine, vol. 4, no. 3(2009), pp. 16-21.

[2] http://news.sina.com.cn/c/2012-11-08/043925533283.shtml.

[3] http://news.xinhuanet.com/legal/2012-11/08/c_123927686.htm.

[4] L. Jian, L. Xiaodong and Q. Y. Jiang, "The CBTC District Control Center Subsystem in Urban Mass Transit”, Modern Urban Transit, no. 2, (2012), pp. 1-6.

[5] State Radio Regulation of China, "On the adjustment of the $2.4 \mathrm{GHz}$ band transmit power limits and related issues", (2002).

[6] Ministry of Industry and Information Technology of the People's Republic of China, "Micro-power (short-range) radio equipment management Interim Provisions", (1998).

[7] L. Ming, H. S. Wang and Y. Fan, "Test and Analysis on the Interference from Wi-Fi Signals to the CBTC Systems", Applied Mechanics and Materials. Switzerland: Trans Tech Publications, (2014), pp. 543-547.

[8] Z. Ying, "Study on CBTC based on Wireless Communication", Communications Technology, no. 12, (2011), pp. 137-141.

[9] A. Y. Yang, "Engineering Applications of Wireless Communication Transmission on Metro CBTC ", Railway Signalling \& Communication Engineering, vol. 9, no. 4, (2012), pp. 51-53.

[10] X. L. Zheng, M. Lin and T. Tao, "The CBTC District Control Center Subsystem in Urban Mass Transit", Computer and communications, vol. 23, no. 3, (2005), pp. 1-6.

[11] L. Zhu, F. R. Yu, B. Ning and T. Tang, "Hand off Management in Communication-Based Train Control Networks Using Stream Control Transmission Protocol and IEEE 802”, 11p WLANs”, EURASIP Journal on Wireless Communications and Networking, (2012).

[12] L. Zhu, F. R. Yu, B. Ning and T. Tang, "Cross-Layer Handoff Design in MIMO-Enabled WLANs for Communication-Based Train Control (CBTC) Systems", Selected Areas in Communications, vol. 30, no. 4, (2012), pp. 719-728.

[13] T. S. Rappaport, “Wireless Communications Principles and Practice”, (2006). 